\title{
Percutaneous vertebroplasty in vertebral osteonecrosis (Kümmell's spondylitis)
}

\author{
Huy M. Do, M.D., Mary E. Jensen, M.D., William F. Marx, M.D., and David F. Kallmes, M.D. \\ Section of Interventional Neuroradiology, Department of Radiology, University of Virginia Health \\ Sciences Center, Charlottesville, Virginia
}

The authors report the clinical symptoms and response to therapy of a series of patients who presented with subacute or chronic back pain due to vertebral osteonecrosis (Kümmell's spondylitis) and who underwent percutaneous vertebroplasty.

The authors performed a retrospective chart review of a series of 95 patients in whom 149 painful, nonneoplastic compression fractures were demonstrated and who were treated with percutaneous transpediculate polymethylmethacrylate (PMMA) vertebroplasty. In six of these patients there was evidence of vertebral osteonecrosis, as evidenced by the presence of an intravertebral vacuum cleft on radiography or by intravertebral fluid on magnetic resonance (MR) imaging. Clinical and radiological findings on presentation were noted. Technical aspects of the vertebroplasty technique were compiled. Response to therapy, defined as qualitative change in pain severity and change in level of activity, was noted immediately following the procedure and at various periods on follow-up reviews.

One man and five women, who ranged in age from 72 to 90 years (mean 81 years), were treated. Each patient had one compression fracture. The fractures were at T-11 (one patient), L-1 (two patients), L-3 (two patients), and L-4 (one patient). The pain pattern was described as severe and localized to the affected vertebra, and sometimes radiated along either flank. Pain duration ranged from 2 to 12 weeks, and the pain was refractory to conservative therapy that consisted of bedrest, analgesics, and external bracing. At the time of treatment, all patients were bedridden because of severe back pain. In all patients either plain radiographic or computerized tomography evidence of intravertebral vacuum cleft or MR imaging evidence of vertebral fluid collection consistent with avascular necrosis of the vertebral body was demonstrated. Four patients underwent bilateral transpediculate vertebroplasty, and two patients underwent unilateral transpediculate vertebroplasty. The fracture cavities were specifically targeted for PMMA injection. Additional fortification of the osteoporotic vertebral body trabeculae was also performed when feasible. "Cavitygrams" or intraosseous venograms with gentle contrast injection were obtained prior to application of cement mixture. In all patients subjective improvement in pain and increased mobility were demonstrated posttreatment. The follow-up period ranged from 4 to 24 hours after treatment. Two patients made additional office visits at 1 and 3 months, respectively. 
Patients presenting with vertebral osteonecrosis (Kümmell's spondylitis) often suffer from local paraspinous or referred pain. When performing vertebroplasty on these patients, confirmation of entry into the fracture cavities with contrast-enhanced "cavitygrams" should be performed prior to injection of PMMA cement. The response to vertebroplasty with regard to amelioration of pain and improved mobility is encouraging.

\section{Key Words * vertebroplasty * vertebral osteonecrosis * Kümmell's spondylitis * methylmethacrylate}

Percutaneous vertebroplasty has been proposed as a treatment for achieving pain palliation in patients who present with vertebral compression fractures resulting from both nonneoplastic and neoplastic disease.[1] In properly selected patients, lasting pain relief may be achieved in those who undergo polymethylmethacrylate (PMMA) vertebroplasty.[5] In a small subset of patients who present with painful vertebral compression fractures radiography may also demonstrate vertebral osteonecrosis. In this subset of patients in our study there was no difference in demographic characteristics when compared with the main group of patients treated, including sex, age, and duration of pain. In general, patients in this subset tend to be elderly women with painful, nonneoplastic osteoporotic compression fractures of the lower thoracic or lumbar spine that were refractory to conservative management. We report a series of patients who presented with subacute or chronic pain in the setting of vertebral osteonecrosis, and we detail the clinical presentation and response to therapy after undergoing percutaneous vertebroplasty.

\section{CLINICAL MATERIAL AND METHODS}

Over the past 5 years, 95 patients with 149 painful vertebral body compression fractures were treated with percutaneous vertebroplasty at our institution. Of these, six patients $(6.3 \%)$ had a medical history and neuroimaging findings that were consistent with Kümmell's spondylitis. The medical and radiological records obtained in these patients were retrospectively reviewed.

\section{RESULTS}

Six patients with six painful vertebral body compression fractures (one at T-11, two at L-1, two at L-3, and one at L-4) were evaluated. Patient age ranged from 72 to 90 years (mean 81 years). There were five women and one man. All patients had a medical history, physical, and radiological evidence consistent with Kümmell's spondylitis. All patients had generalized osteoporosis. In two patients there was either plain radiographic or computerized tomography $(\mathrm{CT})$ evidence of intravertebral vacuum cleft phenomenon (Fig. 1), and in all six patients a finding of a fluid collection was demonstrated on magnetic resonance (MR) imaging (Fig. 2). 


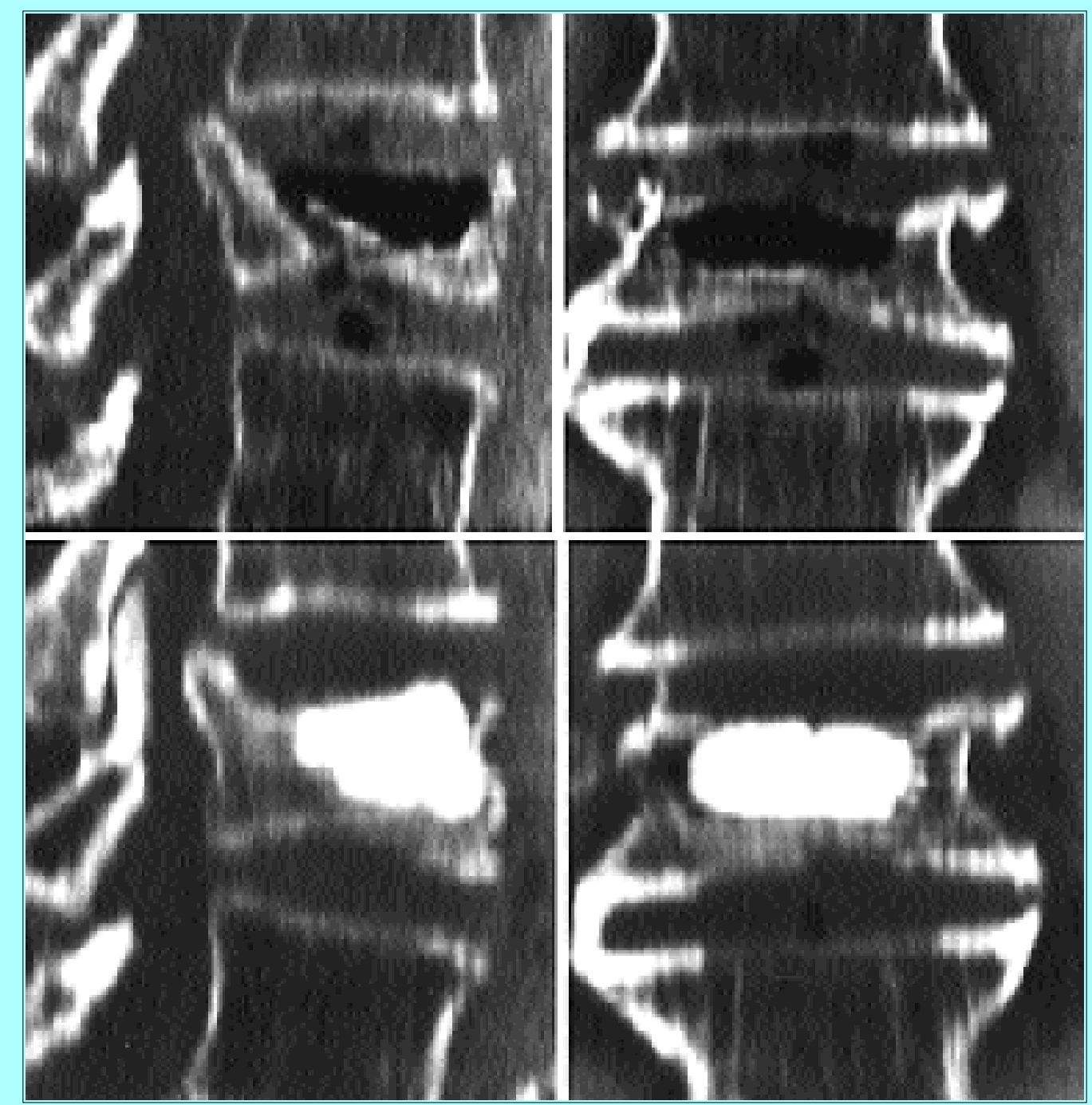

Fig. 1. Representative reformatted CT studies obtained in an 81-year-old woman who had an L-4 compression fracture. Upper Left and Right: Sagittal and coronal reformatted pretreatment CT scans demonstrating the compression deformity with intravertebral gas. Lower Left and Right: Sagittal and coronal reformatted CT scans postvertebroplasty revealing effective filling of fracture cavity with PMMA. 


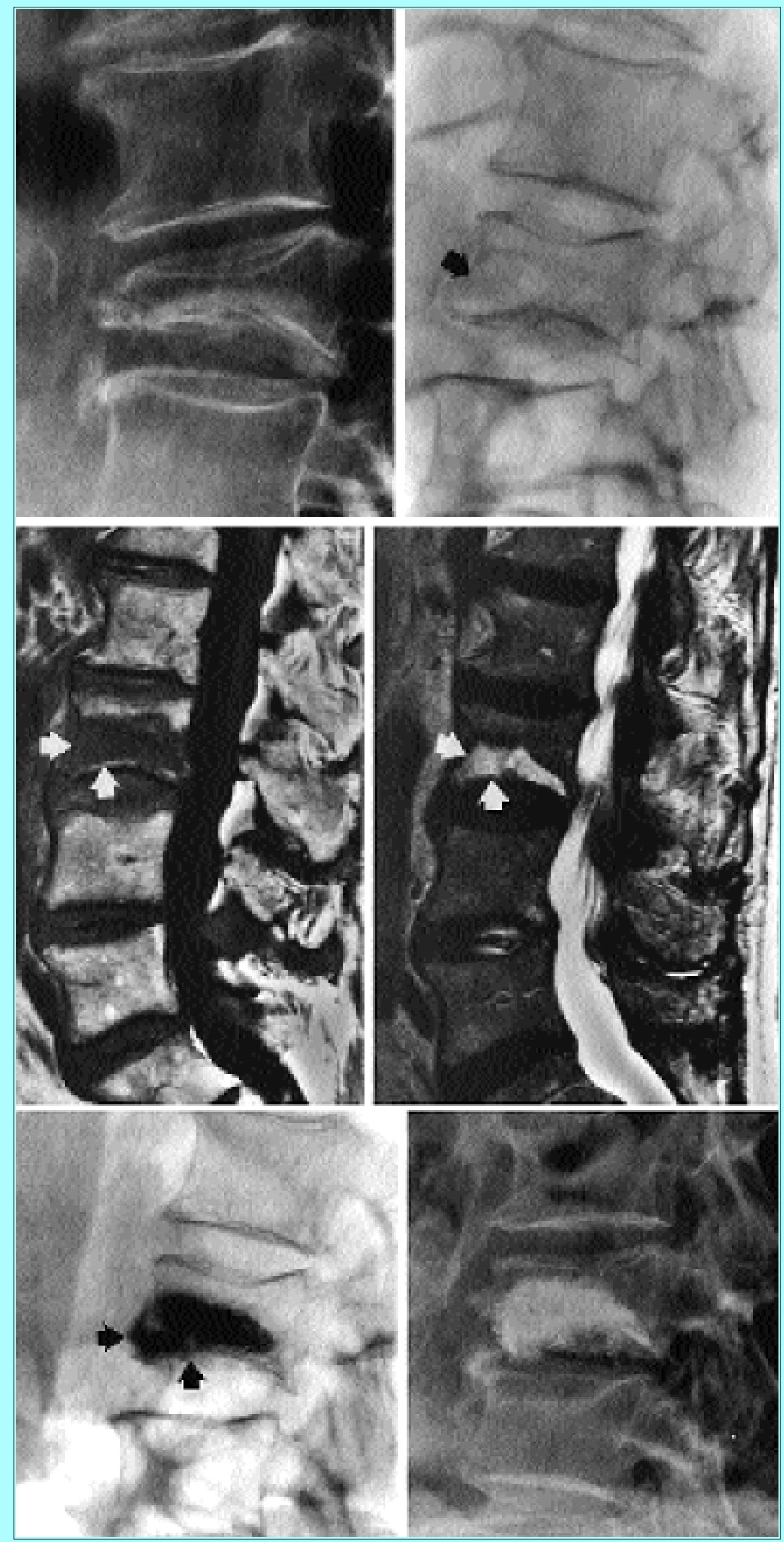


Fig. 2. Imaging studies obtained in a 90-year-old man who had an L-3 compression fracture. Lateral radiographs obtained with the patient in an upright weightbearing (upper left) and prone position (upper right) demonstrating the dynamic unstable nature of the fracture, with expansion of the fracture seen on the film of the non-weightbearing spine (upper right, arrow). Sagittal $\mathrm{T}_{1}$ - (center left) and $\mathrm{T}_{2}$-weighted (center right) $\mathrm{MR}$ images showing the fracture cavity as a discrete area of abnormal low and high signal, respectively (arrows) consistent with vertebral osteonecrosis. Lateral radiographs of the spine in a weightbearing position obtained immediately (lower left) and 1 month (lower right) after vertebroplasty demonstrate stabilization of the fracture.

In all patients a trial of conservative management consisting of rest, analgesics, and/or external back bracing failed to alleviate pain. There was no evidence of infection or malignancy in any patient. Percutaneous vertebroplasty was successfully performed in all patients. Our group[5] has previously described the technique of transpediculate vertebroplasty; its application was the same in this group of patients. The only difference was the aquisition of a "cavitygram," in addition to the usual intraosseous venogram, prior to preparation and deposition of the PMMA cement. This should be performed with very gentle iodinated contrast injection (Fig. 3).

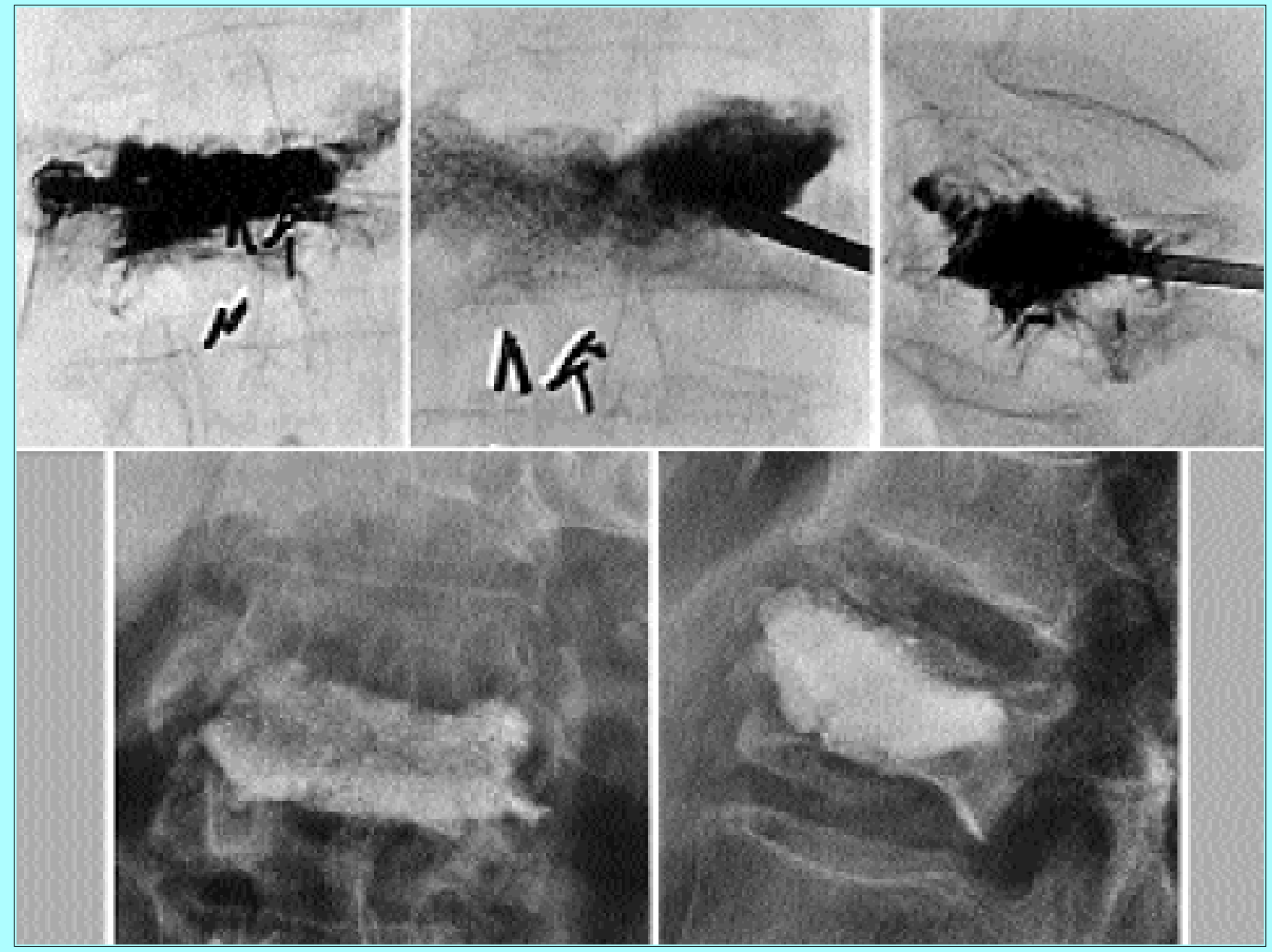

Fig. 3. Imaging studies obtained in a 72-year-old woman who had an L-1 compression fracture. "Cavitygrams" in anteroposterior (upper left and center) and lateral (upper right) views showing primarily opacification of the fracture cavity with minimal filling of the surrounding trabeculae. Anteroposterior (lower left) and lateral (lower right) radiographs showing successful packing of the fracture cavity with methylmethacrylate. 
We stress the importance of a "gentle" injection because in treating our first two patients with vertebral osteonecrosis, contrast medium was injected with the same force required for the intraosseous venography. This caused significant opacification of the fracture cavities that was then quite difficult to clear and required repeated injections and aspirations with normal saline to clear these spaces adequately to see the passage of PMMA cement when vertebroplasty was performed. The average volume of cement deposited was $9 \mathrm{ml}$ (range 5-12 ml). When injected into the fracture cavities, the cement assumed the shape of these cavities without evidence of extravasation into the surrounding marrow spaces or the paravertebral soft tissues, as is sometimes observed in vertebroplasty of the osteoporotic trabeculae. The dynamic nature of the treated fractures (that is, fractures that assumed greater compression with weight bearing) was effectively stabilized when bone cement was deposited into the fracture cavities (Figs. 1 and 2). Posttreatment, all patients experienced significant subjective pain relief, with documented decrease or cessation of analgesic drug use and increased level of mobility.

\section{DISCUSSION}

Patients with vertebral osteonecrosis often present with pain. This pain is similar to that in patients with the usual nonneoplastic osteoporotic compression fractures that we more frequently see and treat (that is, pain that is severe and localized to the injured vertebra, sometimes radiating to one or both flanks). Vertebroplasty in the treatment of these patients is feasible and in our limited experience, has proved to be efficacious. Our patients' pain and impaired mobility improved after treatment. In addition, the instability of the affected vertebra was successfully managed by introducing PMMA into the fracture cavities.

As related by Van Eenemaam and el-Khoury, in 1891 Hermann Kümmell described five cases of delayed posttraumatic vertebral body collapse after patients sustained a relatively minor traumatic injury.[10] He described painful kyphosis and gibbus formation in these patients. Schmorl, et al.,[9] later described the histopathology of this entity and suggested that some cases are ischemic in origin. In 1978, Maldague, et al.,[6] described the presence of a vacuum cleft within vertebral fractures and suggested that this finding represented focal bone ischemia associated with nonhealing vertebral collapse. The vacuum cleft was noted to appear on extension views, but it became poorly demonstrated with more kyphotic positioning. The majority of patients with this entity have been reported to have predisposing factors for avascular necrosis (that is, chronic corticosteroid use or radiation therapy).[1,6-8,10] Recently, the MR imaging characteristics of avascular necrosis of the vertebral body have been described.[1,3,8] These authors described discrete fluid collections within the area of the vacuum cleft that were quite hypointense on $\mathrm{T}_{1}$-weighted and markedly hyperintense on $\mathrm{T}_{2}$-weighted $\mathrm{MR}$ images. These fluid collections lack adjacent areas of inflammatory changes and soft-tissue masses, which are often seen in cases of osteomyelitis and abscess. The high signal intensity demonstrated on $\mathrm{T}_{2}$-weighted $\mathrm{MR}$ images in the setting of osteomyelitis and malignant neoplasms is more diffuse and less intense than that in patients with avascular necrosis.[8] Zones of diminished signal surround these horizontal areas of $\mathrm{T}_{2}$ hyperintensities. This so-called "double-line sign" has been characterized as a peripheral band of sclerosis that surrounds a central area of granulation tissue in both avascular necrosis of the vertebral body and femoral head.[8] Dupuy, et al.,[3] have evaluated the histological characteristics of these fluid collections and found no evidence of infection or malignancy. They postulated that in certain cases osteoporotic vertebral body compression fractures with complicating avascular necrosis lead to the development of fluid collections. 
In two of our patients, we were able to document the unstable nature of their compression fractures, as evidenced by worsening of the wedge compression with the patient in the upright position and relief of the collapse while in the supine or prone position (Fig. 2). Percutaneous vertebroplasty has proved to be effective in the treatment of osteoporotic compression fractures, multiple myeloma, metastases, and painful hemangiomas. $[2,4,5]$ This technique works by augmenting the weak osteopenic vertebrae and thus preventing further microfractures and associated pain. Presumably, the intense exothermic effect of the polymerization process of the deposited PMMA may damage the nerve endings of the vertebral endosteum, thereby imparting a loss of sensation to the treated vertebral body. Depositing the cement into the fracture cavity to stabilize and fixate the fluctuating degree of compression is also desirable. In cases in which focal fracture cavities are detected on imaging studies, care must be taken not to inject contrast material too vigorously in an attempt to attain good opacification of the vertebral trabeculae so as not to fill the cavity completely with contrast material. This makes clearing of the opacified cavity quite arduous and time consuming. It is self-evident that any residual contrast material within the fracture cavity would render visualization of the cement mixture (which itself is opacified with barium sulfate and tungsten) somewhat difficult. Further follow-up review, however, is warranted to study the long-term effect of PMMA in these fracture cavities.

This study is somewhat limited by the small number of patients. The length of follow up is also short. Continued accumulation of patients and follow-up data will be helpful for further validation of this technique.

\section{CONCLUSIONS}

Percutaneous transpediculate vertebroplasty is efficacious in the treatment of Kümmell's spondylitis. The dynamic nature of these fractures is effectively stabilized with injection of bone cement into the fracture cavities.

\section{References}

1. Bhalla S, Reinus WR: The linear intravertebral vacuum: a sign of benign vertebral collapse. AJR 170:1563-1569, 1998

2. Cotten A, Boutry N, Cortet B, et al: Percutaneous vertebroplasty: state of the art. Radiographics 18:311-323, 1998

3. Dupuy DE, Palmer WE, Rosenthal DI: Vertebral fluid collection associated with vertebral collapse. AJR 167:1535-1538, 1996

4. Gangi A, Dietemann J, Schultz A, et al: Interventional radiologic procedures with CT guidance in cancer pain management. Radiographics 16:1289-1306, 1996

5. Jensen ME, Evans AJ, Mathis JM, et al: Percutaneous polymethylmethacrylate vertebroplasty in the treatment of osteoporotic vertebral body compression fractures: technical aspects. AJNR 18:1897-1904, 1997

6. Maldague BE, Noel HM, Malghem JJ: The intravertebral vacuum cleft: a sign of ischemic vertebral collapse. Radiology 129:23-29, 1978

7. Malghem J, Maldague B, Labaisse MA, et al: Intravertebral vacuum cleft: changes in content after 
supine positioning. Radiology 187:483-487, 1993

8. Naul LG, Peet GJ, Maupin MB: Avascular necrosis of the vertebral body: MR imaging. Radiology 172:219-222, 1989

9. Schmorl G, Besemann EF, Junghanns H: The Human Spine in Health and Disease, ed 2. New York: Grune \& Stratton, 1971, pp 268-269

10. Van Eenemaam DP, el-Khoury GY: Delayed post-traumatic vertebral collapse (Kummell's disease): case report with serial radiographs, computed tomographic scans, and bone scans. Spine 18:1236-1241, 1993

Manuscript received March 23, 1999.

Accepted in final form June 14, 1999.

Address reprint requests to: Huy M. Do, M.D., Neuroradiology, S-047, Department of Radiology, Stanford University Medical Center, Stanford, California 94305-5105. email: huymdo@ stanford.edu. 